\title{
Finite Element Modeling of Electromagnetic Signal Propagation in a Phantom Arm
}

\author{
Todd A. Kuiken, Member, IEEE, Nikolay S. Stoykov, Member, IEEE, Milica Popović, Member, IEEE, \\ Madeleine Lowery, Member, IEEE, and Allen Taflove, Fellow, IEEE
}

\begin{abstract}
Improving the control of artificial arms remains a considerable challenge. It may be possible to graft remaining peripheral nerves in an amputated limb to spare muscles in or near the residual limb and use these nerve-muscle grafts as additional myoelectric control signals. This would allow simultaneous control of multiple degrees of freedom (DOF) and could greatly improve the control of artificial limbs. For this technique to be successful, the electromyography (EMG) signals from the nerve-muscle grafts would need to be independent of each other with minimal crosstalk. To study EMG signal propagation and quantify crosstalk, finite element (FE) models were developed in a phantom-arm model. The models were validated with experimental data collected by applying sinusoidal excitations to a phantom-arm model and recording the surface electric potential distribution. There was a very high correlation $(r>0.99)$ between the FEM data and the experimental data, with the error in signal magnitude generally less than $5 \%$. Simulations were then performed using muscle dielectric properties with static, complex, and full electromagnetic solvers. The results indicate that significant displacement currents can develop $(>50 \%$ of total current) and that the fall-off of surface signal power varies with how the signal source is modeled.
\end{abstract}

Index Terms-Control, electromyography (EMG), finite element (FE), modeling, prosthesis.

\section{INTRODUCTION}

$\mathbf{I}$ MPROVING the control of artificial arms remains a considerable challenge. The function of current myoelectric prostheses is limited to control of a single motor or motion at a time. For example, the transhumeral amputee cannot operate the prosthetic hand, wrist, and elbow at the same time with current myoelectric prostheses.

Although the limb is lost with an amputation, the control signals to the limb remain in the residual peripheral nerves of the amputated limb. The potential exists to tap into these lost con-

Manuscript received April 1, 2001; revised December 1, 2001. This work was supported by a Biomedical Engineering Research Grant from the Whitaker Foundation, the National Institute of Child and Human Development under Grant 1K08HD01224-01A1, and the National Institute of Disability and Rehabilitation Research under Grant H133G990074-00.

T. A. Kuiken is with the Rehabilitation Institute of Chicago, Deptartment of Physical Medicine and Rehabilitation, Northwestern University Medical School, Chicago, IL 60204 USA and also with the Electrical and Computer Engineering Deptartment, Northwestern University, Evanston, IL 60204 USA.

N. S. Stoykov and M. Lowery are with the Rehabilitation Institute of Chicago, Chicago IL 60611 USA and also with the Department of Physical Medicine and Rehabilitation, Northwestern University Medical School, Chicago, IL 60204 USA.

M. Popović is with the Department of Electrical and Computer Engineering, McGill University, Montreal, QC, H3A 2K6, Canada.

A. Taflove is with the Dept. of Electrical and Computer Engineering, Northwestern University, Evanston, IL 60204 USA.

Publisher Item Identifier S 1534-4320(01)11409-9.

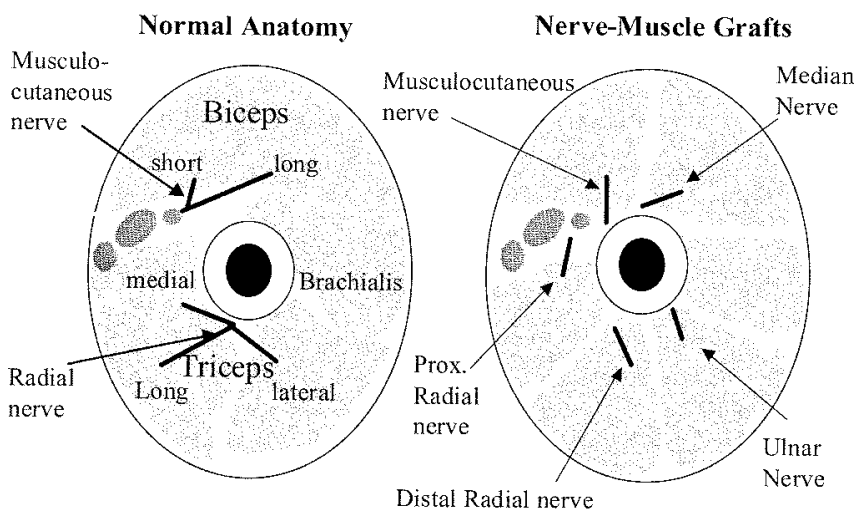

Fig. 1. The use of nerve-muscle grafts for additional myoelectric control sites in a transhumeral amputee.

trol signals using nerve-muscle grafts and greatly improve the control of externally powered prostheses. It may be possible to denervate expendable regions of muscle in or near an amputated limb and graft the residual peripheral nerve stumps to these muscles [1]. The nerves would reinnervate these muscles. Then, the surface electromyograms (EMGs) from the nerve-muscle grafts might be used as additional myoelectric control signals for an externally powered prosthesis. The main advantages of a nerve-muscle graft system are that there are potentially more discrete myoelectric control signals available and that these signals relate directly to the original function of the limb.

For the long transhumeral amputee one head of the biceps and two heads of the triceps would be denervated (see Fig. 1). The median, ulnar, and distal radial nerves would be grafted onto these heads and allowed to reinnervate these regions of muscle. These nerve-muscle grafts would produce five EMG control signals that could be used for simultaneous control of at least the terminal device and elbow and possibly a third degree of freedom (DOF), such as a wrist rotation or wrist flexion-extension. Furthermore, shoulder motion would still be available to control additional degrees of freedom.

For this technique to be successful, the surface EMGs recorded over each of the nerve-muscle grafts must be relatively independent of each other. Crosstalk between the nerve-muscle grafts could be problematic. For the clinical application of myoelectric prostheses, setting a threshold prevents crosstalk from interfering with prosthesis operation. The amputee must generate an EMG signal greater than the threshold to operate the prosthesis. The threshold is set above the baseline noise and crosstalk from nearby muscles. No published data is available on what crosstalk levels are acceptable for myoelectric prostheses, but, clearly, the greater the 
crosstalk, the higher the threshold must be set and the harder it is for the amputee to operate the myoelectric prosthesis.

With the nerve-muscle graft technique, nerves would be grafted onto individual heads of the biceps and triceps in a transhumeral amputee. Therefore, crosstalk between these heads would be a critical factor. Crosstalk has not been measured between the biceps and triceps, far less between the individual heads of these muscles. However, crosstalk has been measured between recording sites over muscles in the human leg. De Luca and Merletti [2] selectively stimulated the tibialis anterior muscle in 12 normal human subjects while recording the surface EMG over the tibialis anterior muscle, the peroneus brevis muscle, the soleus muscle, and the tibial bone. They reported root mean square (rms) crosstalk values of $6.2 \%, 5.0 \%$, and $18.4 \%$, respectively. They suggested that the larger signals over the tibial bone were due to the close proximity of the recording site and the relatively low conductivity of the bone. In larger people, with more soft tissue over the bone, the tibial signals were greater. In the smallest person, with little soft tissue over the bone, the tibial signal was smaller than the signal recorded over the peroneus brevis. This is an excellent example of how the anatomy of the limb can affect the EMG field distribution and crosstalk. Koh and Grabiner [3] measured crosstalk over the hamstrings with quadriceps femoris activation. Crosstalk averaged $17.1 \%$ over the lateral hamstrings and $11.3 \%$ over the medial hamstrings with conventional bipolar recordings (8-mm electrodes spaced 20-mm apart). Crosstalk decreased to $7.6 \%$ over the lateral hamstrings and $4.2 \%$ over the medial hamstrings using simulated double differential recordings.

It is possible that the crosstalk between the proposed nervemuscle grafts will be problematic. Each head for the biceps and triceps is relatively small, and they are all in close proximity to each other. This is especially true in an amputee, where some muscles may be partially amputated and all muscles are atrophied. If crosstalk between nerve-muscle grafts is problematic, it may be possible to decrease crosstalk and increase signal independence with surgical manipulation. Removing subcutaneous fat or thinning the skin could increase signal power. Placing insulating tissues, such as fat, or an insulating biomaterial, such as silicone, between muscle grafts might decrease current spread and reduce crosstalk. Placing an electric shield, such as a wire mesh, between the muscles may also decrease crosstalk.

To explore the idea of using nerve-muscle grafts and to test ways for increasing myoelectric signal independence, a tool is need to study electromagnetic signal propagation in biologic tissues. In this paper, finite element (FE) models of electromagnetic signal propagation in a phantom limb are presented. The technique is validated with experimental data. The finite-element method (FEM) is appropriate for many reasons. It is appropriate for low frequency content of the EMG. It is a flexible tool that allows the modeling of any geometry and can incorporate multiple tissues and/or materials. Anisotropic materials can be modeled. Analysis can be done both in the frequency domain and the time domain. The FEM also allows easy viewing of the entire electric potential field distribution, current densities, or any other desired electromagnetic parameters.

Many other sophisticated models have been developed to study EMG. Most of these studies have used infinite space geom- etry or infinite plane geometry [4], [5]-[13], [23]. Some studies have considered cylindrical volumes embedded in infinite volume conductors [14], [15]. Only a few studies have considered the finite dimensions of a cylindrical volume conductor [16]-[20]. These studies have shown that the boundary can have a significant impact on single fiber action potentials. The FEM allows modeling of any shape. Therefore, we can study both cylindrical models and, eventually, more complex and realistic geometries and multilayer compartment models, we hope.

The electrical permittivities of many biological tissues are very high, especially for muscle and skin [21]-[25]. Thus, there is the potential to develop significant displacement currents even at the relatively low frequencies found in bioelectric signals. This could significantly affect the amplitude, as well as the phase and shape, of the EMG signal. Although some investigators have considered the effects of capacitance near the source [10], [20], [26], [27], none have included the permittivity of the tissue as a whole. A full electromagnetics model is necessary to quantify these effects and determine their significance. An important property of the FEM is that it allows full Maxwell's equations to be used and, thus, includes the capacitive properties of tissues.

How the electrical sources or action potentials are modeled is very important to fall-off of the electric field with increasing distance from the source. Some researchers have modeled the action potential as a simple dipole [4], [15], and many groups have used a balanced tripole [5], [8], [9], [11], [19], [28], whereas others [6], [7], [13], [14], [20], [29] have used the more realistic analytic waveform described by Rosenfalk [30] and modified by Nandedkar and Stalberg [31]. The electric field will fall off more rapidly with a balanced tripole than with a dipole, resulting in less crosstalk around the limb. The real action potential is neither a dipole nor a tripole, but a source with a more complex waveform. It is unknown how fast the electric field of such a waveform will decay in space.

Finally, most EMG models previously presented focused on the shape of the action potential recorded directly over the source and did not consider surface potential around the limb, or the fall-off in signal power. Merletti et al. [11] quantified fall-off of signal power (average rectified value), but this was limited to models with a recording site over the source using an infinite hemispace model. Roeleveld et al. [19], [32], [33] measured and modeled EMG signal propagation around a limb; however, their focus was on waveform shape and they did not quantify signal power. Finally, Winter et al. [15] used an infinite space model, very small limb geometry, and dipole sources, which limits the application of the model. For our clinical application, the fall-off of EMG signal power around the limb and crosstalk are critical issues that need a thorough evaluation.

In this study, we have developed finite element electromagnetics models of EMG signal propagation in the arm to predict surface potential distributions. These FE models are validated with data recorded in experiments using a phantom-arm model. Then, we present simulations using human muscle dielectric properties to gain insight on the usefulness of this method and the importance of using full Maxwell's equations with these models. 


\section{METHODS}

\section{A. Experimental Methods}

We have started by studying electromagnetic signal propagation in a phantom-arm model. The phantom arm model consists of a cylinder of finely ground cooked meat $30-\mathrm{cm}$ long with a diameter of $10 \mathrm{~cm}$. This phantom model has a number of advantages. It is a consistent biological model that is made of the dominant tissue of the arm and has dielectric properties similar to those of human muscle. It is a homogeneous and isotropic material that simplifies the initial modeling. It has a regular, repeatable, and symmetrical shape that is the approximate size of the human arm. It is an easy model to work with and is relatively inexpensive. Sinusoidal sources can be applied that eliminate the effect of dispersion (the variation of dielectric properties as a function of electromagnetic field frequency). Finally, it minimizes the number of in vivo animal and human experiments that will be required.

An array of 12 bipolar electrodes was placed on and in the phantom. Eight electrode pairs were evenly placed at $45^{\circ}$ intervals around the surface in the middle of the model. The poles of these electrodes had a diameter of $6.0 \mathrm{~mm}$ and were separated by $6.0 \mathrm{~cm}$. This relatively large pole separation was chosen to ensure accurate spacing and alignment of the electrodes. The small poles were used to improve the accuracy of the model. Three pairs of deep electrodes were placed in the phantom model. These had spherical poles measuring $4.0 \mathrm{~mm}$ in diameter and insulated 14-gauge wire leading to the poles. These stiff wires were pressed into the side of the phantom model a specified depth. One pair was placed $2.0-\mathrm{cm}$ deep with a pole separation of $6.0 \mathrm{~cm}$ at the middle of the model. The second pair was placed $4.0-\mathrm{cm}$ deep with a pole separation of $6.0 \mathrm{~cm}$ at the middle of the model. The third pair was placed $2.0-\mathrm{cm}$ deep and either end of the model with a pole separation of $24 \mathrm{~cm}$. Finally, the small spherical electrodes were used to apply a surface excitation in the middle of the model with a pole separation of $1.0 \mathrm{~cm}$.

The excitation signals consisted of a simple sine wave at 150 $\mathrm{Hz}$, a frequency close to the mean of the surface EMG. Potentials at the recording sites were amplified with an Axon Instruments Cyber Amp 360 (Axon Instruments, Inc., Foster City, CA 94404 USA) and were low-pass filtered at $10000 \mathrm{~Hz}$. The input current, the input voltage, and the voltage at each recording site were recorded with a CED 1410 data acquisition system (Cambridge Electronic Design, Cambridge, CB4 oFE, U.K.) using a sampling frequency of $5000 \mathrm{~Hz}$. The excitation signals were sequentially applied to a $6.0-\mathrm{cm}$-spaced surface electrode pair, the 1.0 -cm-spaced surface electrodes, the 2.0 -cm-deep middle electrodes, and the 4.0-cm-deep middle electrodes. The experiment was repeated five times.

At the beginning and end of each experiment, the input signals were applied to each end of the model through copper plate electrodes covering the end of the model. In this manner, current was passed through the entire cylindrical volume of the model. This allowed calibration of the experimental setup because the potential should be the same when measured by any electrodes with the same pole separation. This also allowed measurement of the tissue conductivity using the total current and recorded potential over a given distance. The conductivity $\sigma$ was calculated from the expression for the conductance of a cylinder $G=$ $\sigma A / l$ where $G$ is the conductance and $A$ and $l$ are the cross-sectional area and the length of the cylinder, respectively. With the conductance expressed as the ratio of current $(I)$ and voltage drop $(V)$ over the cylinder, we finally obtain the equation $\sigma=$ $I l / A V$. The permittivity of the tissue was measured at a frequency of $150 \mathrm{~Hz}$ with an HP-4194A impedance-gain-phase analyzer (Hewlett-Packard, Palo Alto, CA 94304 USA).

\section{B. FE Modeling}

The finite element analysis software used was EMAS by the Ansoft Corporation(Ansoft, Pittsburgh, PA 15219-1119 USA). EMAS is a robust FE analysis system with efficient geometry modeling. It allows frequency-domain and time-domain analysis with a full range of field solvers. Frequency-domain analysis was used in these simulations to eliminate any effects of dispersion.

In the FE models, both linear and quadratic tetrahedral elements were tested. We found that linear elements were more robust with respect to distortion than were quadratic elements. Thus, the mesh generation with linear elements was easier.

The geometry of the FE model replicated the physical model (see Fig. 2); however, it was not necessary to include the wires. Both linear tetrahedral and quadratic tetrahedral elements were tested. FE models yield only an approximation to the exact solution of the underlying boundary value problem. Accuracy depends on the size and order of the finite elements used. To assess this accuracy, a convergence analysis was performed based on eight differently sized models. Both the overall number of elements and their distribution within the model are important. Fine discretization of regions with high intensity of the electric field is essential. At least 10 elements were used between each pair of electrodes. The mean conductivity and permittivity measured above were used in these simulations.

Two different boundary conditions were tested, a spherical open boundary (an absorbing boundary condition) and an insulated electric wall (a grounded metal box containment with a $2.0-\mathrm{cm}$ air buffer placed around the phantom model). No difference in results was observed. Because the electric wall allowed for fewer air elements and more efficient modeling, it was used.

Both point sources and excitations distributed over the surface of the electrodes were tested. They produced equivalent results. Distributed excitations were used since this emulated the physical model. A sinusoidal $150-\mathrm{Hz}$ excitation of $1.0 \mathrm{~mA}$ was applied to both electrodes. FE models were made to simulate all of the different experimental paradigms including surface sources and deep sources.

In order to assess the possible contribution of displacement currents and eddy currents to the human EMG, additional simulations were performed using the dielectric properties of human muscle. The conductivity $(\sigma)$ and relative permittivity $\left(\varepsilon_{r}\right)$ for muscle at $150 \mathrm{~Hz}$ as measured by Gabriel et al. [24] were used $\left(\sigma=0.2 \mathrm{~S} / \mathrm{m}, \varepsilon_{r}=1.50 \times 10^{7}\right)$. The source was placed $1.0-\mathrm{cm}$ deep with a $1.0-\mathrm{cm}$ spacing between the excitation electrodes. Three different frequency domain approaches were used to calculate the electromagnetic fields. In the first one, the electric 


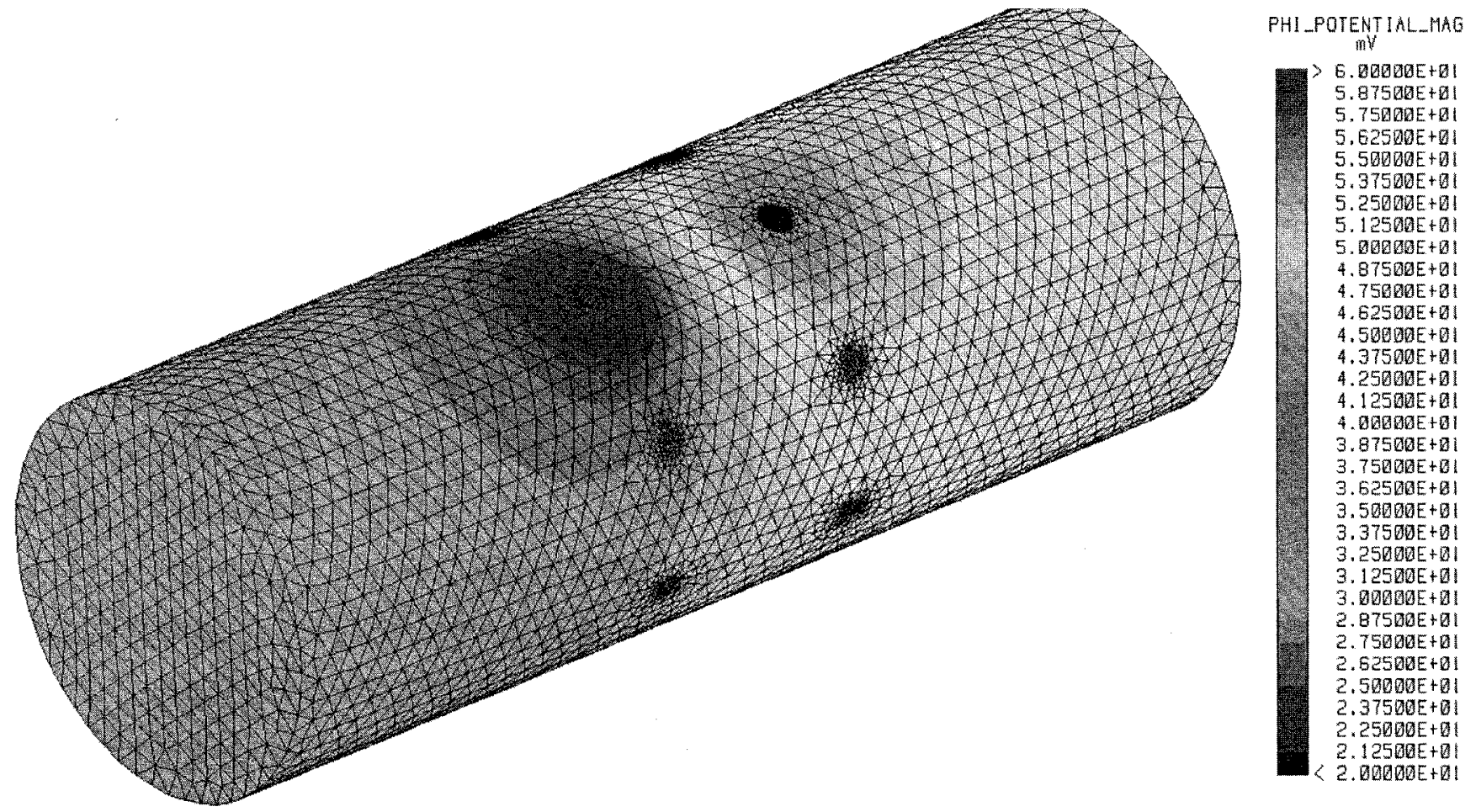

Fig. 2. Surface electric potential distribution with FE model of phantom arm. The excitation source was applied to the surface with $6.0-\mathrm{cm}$ pole separation. There are eight bipolar surface recordings with $6.0-\mathrm{cm}$ pole separation.

scalar potential was obtained by solving the Laplace equation with real material properties. This corresponds to a stationary field in purely resistive media. In the second approach, complex electric properties were used, taking into account the permittivity of the media. A gradient (curl free) electric field was still assumed and, thus, any possible influence of the magnetic field on the results was disregarded. In the third approach, based on the full Maxwell's equations, the complete electromagnetic field was resolved.

Finally, simulations were done to study the difference in signal fall-off and crosstalk when the EMG source is modeled with a dipole, tripole, or realistic waveform. For these simulations, the model was $30-\mathrm{cm}$ long with a fine square surface mesh, and a 1.0-mA source was placed 1.0-cm deep. For the dipole source, a physiologic pole separation of $2.1 \mathrm{~mm}$ was used. For the tripole, the separation between the first and second poles was also $2.1 \mathrm{~mm}$ and the separation between the second and third poles was $4.8 \mathrm{~mm}$ for a total of $6.9 \mathrm{~mm}$ [11], [18]. For the realistic waveform, the analytic expression described by Rosenfalk [30] was applied to the surface of a cylinder with a diameter of $0.1 \mathrm{~mm}$ (the approximate diameter of a muscle fiber) and a length of $15 \mathrm{~mm}$. The EMG contains a broad frequency content, muscle is highly dispersive (eg. permittivity varies greatly with frequency), and the FE method currently is unable to model dispersive media. Therefore, it was necessary to ignore permittivity in these models and perform quasistatic simulations. The conductivity of muscle from Gabriel et al. at $150 \mathrm{~Hz}$ was used. Analysis was again done in the frequency domain for computational efficiency.

The surface EMG waveforms were generated circumferentially around the model by acquiring the surface electric po- tential distribution in a line running down the entire length of the model (parallel to the direction of the muscle fiber). This correlates to a monophasic recording of an EMG signal propagating under an electrode. The rms value of these waveforms represents the inherent signal power at the surface around the model.

Bipolar recordings are more selective, thus minimizing crosstalk, and are generally used for surface EMG recordings. Bipolar electrodes in myoelectric prostheses typically have about $2.0 \mathrm{~cm}$ of separation between the poles. To model this clinical scenario, the bipolar recordings were simulated by taking the difference in the surface electric potential map between points separated longitudinally by $2.0 \mathrm{~cm}$. This process was applied down the entire length of the model to emulate an EMG signal propagating under bipolar electrodes.

\section{RESULTS}

The conductivity of the experimental tissue measured before each experiment was $1.35 \pm 0.05 \mathrm{~S} / \mathrm{m}$ and it increased slightly during the experiment ( $\sigma=1.44 \pm 0.01$, a $7 \%$ increase). This increase was likely secondary to warming of the model. The conductivity was slightly higher (1\%-3\%) when measured with the deep electrodes as opposed to the surface electrodes. This was probably due to a slight water loss from the superficial tissue raising the resistivity of the tissue near the surface. The overall average conductivity of the phantom tissue used for the finite element models was $1.39 \mathrm{~S} / \mathrm{m}$. This conductivity is several times higher than that of regular muscle due to the salt that was added as a preservative. The relative permittivity $\varepsilon_{r}$ of the tissue was measured to be $3.29 \times 10^{7}$ at $150 \mathrm{~Hz}$. This is also higher than that of regular muscle. 
TABLE I

Phantom-Limb Measured Electric Potential Field Compared to FEM Calculations. A Surface Excitation Was Used With 6.0-Cm Spacing Between Excitation Electrodes. All Potentials Are Divided by the InPUt CurRent to Normalize Values For Comparison

\begin{tabular}{c|c|c|c}
\hline Channel & $\begin{array}{c}\text { Normalized } \\
\text { Measured Potentials } \\
(\mathrm{mV}) \\
(\text { Mean } \pm \text { Std })\end{array}$ & $\begin{array}{c}\text { Normalized } \\
\text { Calculated } \\
\text { Potentials } \\
(\mathrm{mV})\end{array}$ & $\begin{array}{c}\text { Deviation of } \\
\text { Calculated from } \\
\text { Measured Potentials } \\
\text { in \% }\end{array}$ \\
\hline $0^{\circ}$ & NA & NA & NA \\
\hline $45^{\circ}$ & $5.26 \pm 0.29$ & 5.44 & 3.42 \\
\hline $90^{\circ}$ & $2.71 \pm 0.10$ & 2.81 & 3.69 \\
\hline $135^{\circ}$ & $2.14 \pm 0.09$ & 2.19 & 2.33 \\
\hline $180^{\circ}$ & $2.01 \pm 0.10$ & 2.05 & 1.99 \\
\hline $225^{\circ}$ & $2.19 \pm 0.15$ & 2.19 & 0.0 \\
\hline $270^{\circ}$ & $2.70 \pm 0.14$ & 2.80 & 3.70 \\
\hline $315^{\circ}$ & $5.31 \pm 0.38$ & 5.42 & 2.07 \\
\hline $2 \mathrm{~cm} \mathrm{deep}$ & $7.54 \pm 0.45$ & 7.83 & 3.85 \\
\hline $4 \mathrm{~cm} \mathrm{deep}$ & $4.07 \pm 0.40$ & 4.35 & 6.88 \\
\hline $2 \mathrm{~cm}$ & $5.02 \pm 0.22$ & 5.08 & 1.20 \\
\hline poles & & & \\
\hline
\end{tabular}

There are a minimum number of elements needed to discretize a given geometry. In our particular case, this number was about 29000 elements. A model size of about 51000 was sufficient to reach satisfactory accuracy. The difference in the surface electric fields (i.e., a monopolar recording of the EMG) between a 380000 -element model and a 51000 -element model was less than $1.60 \%$. When bipolar recordings with $2.0-\mathrm{cm}$ pole separation were used, the difference between these models was less than $0.3 \%$.

In order to compare the experimental data to the computer simulations, all voltages were normalized with respect to the input current. Results are presented for experimental and simulated data in Table I. Experimental data was consistent over repeated measures; standard deviations of peak voltages were generally less than $10 \%$. The correlation between the measured data and the computer simulations was very high. In all experiments, the correlation coefficient was greater than 0.99. Comparison of absolute magnitudes also showed very good agreement (see Table I). In general, measured potentials were within 5\%-10\% of values predicted by the FE models. The closest agreement was seen when the input signal was applied to the surface with 6-cm pole separation; the calculated potentials were higher than the measured potentials by less than $4 \%$ at the surface sites and $7 \%$ at the deep recording sites. When the input signal was applied to the surface with a $1.0-\mathrm{cm}$ space between the excitation electrodes, the calculated potentials were consistently lower than the measured values, but still quite close. All calculated potentials were within $6.7 \%$ of the mean measured potentials. Similar results were obtained when the input signal was applied to the 2.0-cm-deep electrodes (deviation from measured values $<4.3 \%$, except for the 4.0-cm-deep recording which had a difference of $7.4 \%$ ). When the input signal was applied to the 4.0-cm-deep electrode (simulating deeper muscle fibers), the error increased with differences of $3.5 \%-10.9 \%$. Once again, the calculated values were higher than the measured values.

The surface potential distribution for surface excitation with 1.0-cm pole separation is shown in Fig. 2. As expected, the gen- eral form was concentric equipotential lines. The potential distribution in a cross section through this phantom model at the level of the recording electrodes is shown in Fig. 3. The convexity of the equipotential lines flattens as they move away from the source and reverses on the side opposite of the source. This indicates that the shape of the model is affecting current flow. In unbounded homogenous media, the equipotential lines would be continued concentric circles. With deeper excitation, the surface potentials were diminished and more diffuse.

To assess the potential importance of displacement currents and eddy currents in EMG propagation through muscle, a series of simulations were done using the dielectric properties of human muscle and three different FE solvers. Table II shows the calculated electromagnetic parameters for a single point on surface over the source. The ratio between these parameters is the same at all other points in this single tissue model; only the absolute values vary. The static model estimated a field potential that was $1.7-\mathrm{mV}$ higher than the full electromagnetic (EM) model; this represents an $18.3 \%$ error. Analysis of the current density in the complex model revealed that the peak conduction current and peak displacement current were $84.7 \%$ and $52.9 \%$ of the peak total current, respectively. This yields a ratio of displacement current to conduction current of 0.625 . The corresponding phase difference between current and voltage was $32^{\circ}$.

There was no difference between the conduction currents and the displacement current density distributions calculated by the complex solver and the full EM solver. Furthermore, the magnetic field in the full EM model was very small. This demonstrates that the complex model accurately estimated potential fields and that eddy currents are negligible in EMG propagation through muscle, as expected.

The fall-off of rms surface potential (the intrinsic surface signal amplitude) with different sources is summarized in Fig. 4. The signal amplitude decreases as azimuth around the limb increases for all sources, as expected. Clearly, the fall-off in signal power is slowest with a dipole source. Fall-off of signal power is almost identical with the balanced tripole and analytic wave- 

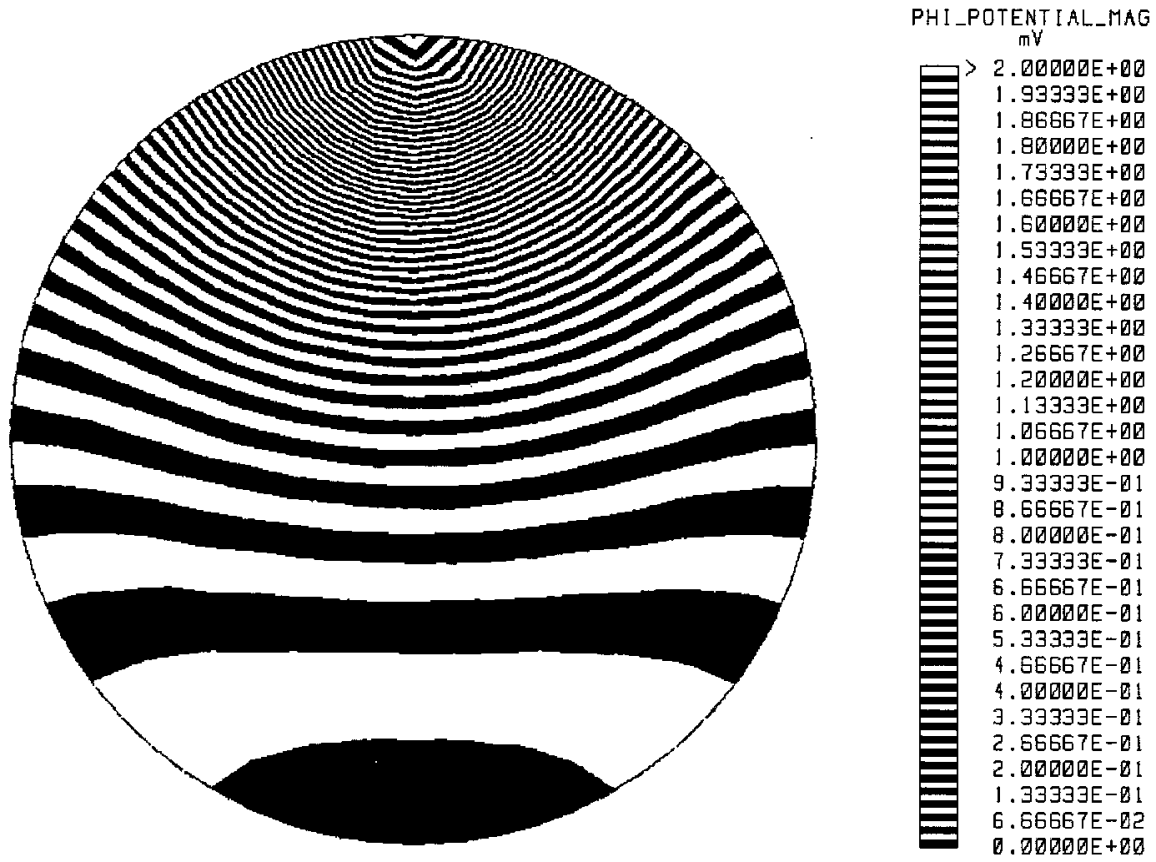

Fig. 3. The electric potential distribution in a cross section through the phantom model at the level of the recording electrodes. The excitation source was applied to the top surface of the model with 1.0 -cm pole separation.

TABLE II

Electromagnetic Parameters Calculated for a Surface Point With Three Different FE Solvers. A 1-mA 150-Hz Source Was Located 1.0 CM Beneath the Surface of the Model. The Material Properties of Muscle Were Used

\begin{tabular}{c|c|c|c|c|c|c}
\hline Solver & $\begin{array}{c}\text { Electric } \\
\text { Potential } \\
(\mathrm{mV})\end{array}$ & $\begin{array}{c}\text { Total } \\
\text { Current } \\
\text { Density } \\
\left(\mathrm{nA} / \mathrm{mm}^{2}\right)\end{array}$ & $\begin{array}{c}\text { Conduction } \\
\text { Current } \\
\text { Density } \\
\left(\mathrm{nA} / \mathrm{mm}^{2}\right)\end{array}$ & $\begin{array}{c}\text { Displacement } \\
\text { Current } \\
\text { Density } \\
\left(\mathrm{nA} / \mathrm{mm}^{2}\right)\end{array}$ & Phase & $\begin{array}{c}\text { Eddy } \\
\text { Current } \\
\text { Density } \\
\left(\mathrm{nA} / \mathrm{mm}^{2}\right)\end{array}$ \\
\hline $\begin{array}{c}\text { Static } \\
\text { Model }\end{array}$ & 10.97 & 6.48 & 6.48 & 0 & $0^{\circ}$ & 0 \\
\hline $\begin{array}{c}\text { Complex } \\
\text { Model }\end{array}$ & 9.27 & 6.48 & 5.49 & 3.43 & $32.0^{\circ}$ & 0 \\
\hline $\begin{array}{c}\text { Full EM } \\
\text { Model }\end{array}$ & 9.27 & 6.48 & 5.49 & 3.43 & $32.0^{\circ}$ & $>0.0001$ \\
\hline
\end{tabular}

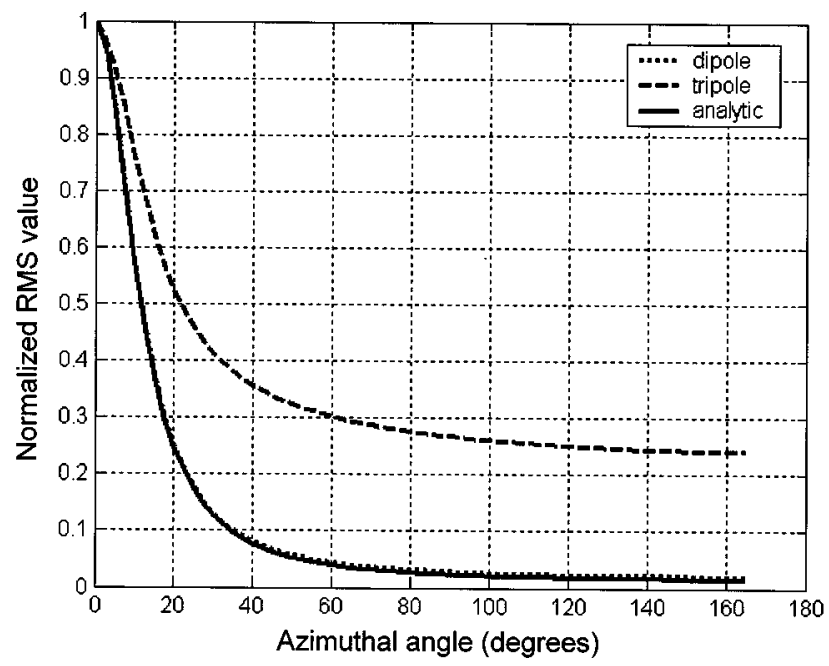

Fig. 4. RMS value of surface electric potential around the limb with source located $10 \mathrm{~mm}$ beneath the surface of the model. form sources; the fall-off is slightly faster with the tripole. When bipolar recordings with $2.0 \mathrm{~cm}$ pole separation are simulated, the fall-off in signal amplitude is faster for all three sources as expected (see Fig. 5). Crosstalk falls to less than $10 \%$ by an azimuth angle of $45.6^{\circ}, 31.1^{\circ}$, and $30.9^{\circ}$ for the dipole, analytic waveform, and tripole, respectively.

\section{DISCUSSION}

The primary goals of this research were to apply finite element method to electromagnetic signal propagation in a limb, to gain an understanding of the FEM parameters, and to validate the FEM with experimental data. We have started with the simplest physical model possible so that we could focus on the simulation technique. Using a perfectly cylindrical single-tissue phantom allowed us to have an intuitive feeling for what the data should look like and allowed us to compare data from the two hemispheres to ensure that it was reasonably accurate by 


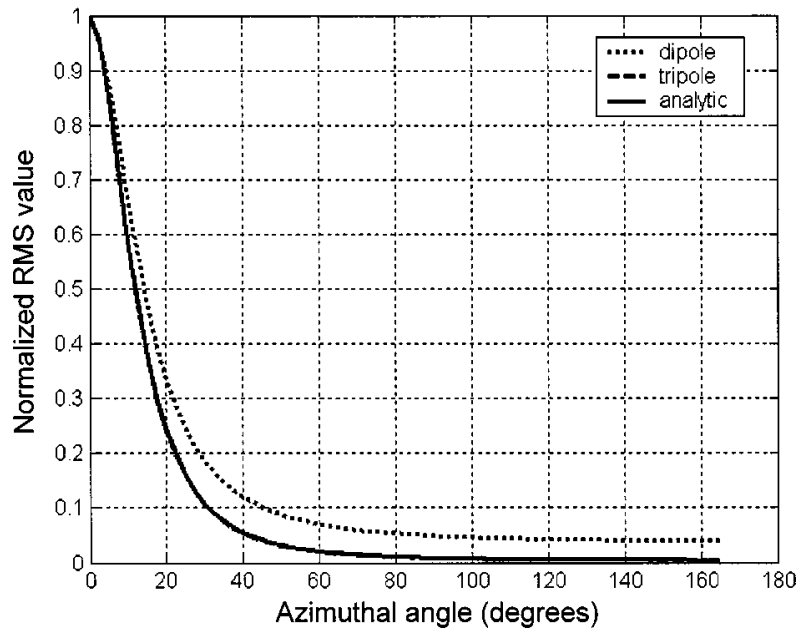

Fig. 5. Surface crosstalk (RMS value) around the limb using bipolar recording with $2.0-\mathrm{cm}$ pole separation. The source is located $10 \mathrm{~mm}$ beneath the surface of the model.

inspection. It also allowed accurate calibration of the experimental setup as well as easy and repeatable measurement of conductivity. By using finely ground meat as the study tissue, we eliminated the issues of anisotropy and tissue inhomogeneity in the phantom. Perhaps the most important simplification was the way in which the sources were modeled. By using stationary sine-wave sources, we are able to do frequency domain analyses that are much simpler and computationally more efficient than time domain analyzes. This also eliminated any error due to dispersion in the model.

Although the model is simple, we feel it is an appropriate start. The model is made out of muscle (albeit processed muscle), the dominant tissue of all limbs. Furthermore, the phantom model is anatomically about the same shape and size as the human arm. The error induced by the commonly used infinite plane or hemispace models [4]-[13], [34] is eliminated.

The conductivity measured for the phantom tissue is about seven times higher than that of normal muscle. This is not surprising, because the meat for the model was salted. The permittivity of the phantom tissue was twice the permittivity that Gabriel et al. [24] reported for muscle. There is a broad range of reported values for muscle permittivity range at these low frequencies $\left(\sim 3.5 \times 10^{5}\right.$ to $\left.1.5 \times 10^{7}\right)$. However, our measured value is above this range and we see no clear reason why the processing of the meat would increase the permittivity. Because the conductivity of the phantom tissue was so high, the relative effect of the phantom tissue permittivity was small.

The correlation between the measured experimental data and the FE model estimations was very high. The correlation coefficients were always $>0.99$, indicating that the geometry of the relative potential distributions were predicted very accurately. The absolute magnitude of the measure potentials was also very close to the FE predicted values. There was generally less than a 5.0\% difference and the calculated values were usually within the standard deviation of the measured values, as well. This indicates that the dielectric constants used in the model were also accurate. There was a somewhat greater difference between the measured and calculated values for the deep electrodes than for the surface electrodes. There are two likely reasons for this difference. The first reason is that there was probably a slight difference in the conductivity of the surface phantom model tissue and deeper tissue. Our measurements indicated that the deeper tissue had a slightly higher conductivity. This may be due to the surface tissue drying out a bit during the experiments, thus lowering the conductivity. The second reason there was a larger difference with the deep-electrode values is that deep-electrode placement was not as accurate as surface electrode place. On the surface, electrodes with a fixed pole separation were used, and it was easy to check measurements. With the deep electrodes, stiff wires were pushed into the phantom model at a set depth. There was undoubtedly some error in the angle of insertion of the wire and, hence, error in the final location of the electrode poles. Overall, the agreement between the experimentally measured potentials and the FE estimation was very high. The use of the finite element method to model electromagnetic signal propagation in a limb was clearly validated.

To date, most EMG models have been quasistatic or stationary models. Only the resistive component of tissue impedence is considered in these static models. This assumes that the capacitive properties of the tissues have no significant effect because the frequency content of bioelectric signal is fairly low. However, the permittivities of biological tissues are generally very high, especially at low frequency. Our model demonstrates the potential importance of the capacitive properties of biological tissues. Using the measured values of conductivity and permittivity from Gabriel et al. [24], it was seen that displacement currents can be $62.5 \%$ as large as conduction currents with a phase of $32^{\circ}$. The resulting electric potential field is $18 \%$ less than what would be estimated with a stationary model-a significant difference. For the sake of completeness, we also looked at the contribution of eddy currents (currents due to the magnetic field). The eddy currents were negligible. Therefore, the complex EM model will be accurate and much more computationally efficient than a full EM model.

The ratio of the magnitudes of displacement current density $J_{\text {disp }}$ and conduction current density $J_{\text {cond }}$ can be predicted with Plonsey's [24] simple equation

$$
\frac{J_{\text {disp }}}{J_{\text {cond }}}=\frac{\omega \varepsilon_{r} \varepsilon_{0}}{\sigma}
$$

where $\omega$ denotes the angular frequency, $\varepsilon_{r}$ is the relative permittivity of the medium, $\varepsilon_{0}=8.854 \times 10^{-12} \mathrm{Fm}^{-1}$ represents the permittivity of vacuum, and $\sigma$ is the conductivity of the medium, measured in $\mathrm{Sm}^{-1}$. At $150 \mathrm{~Hz}$, this value would equal 0.626 , using the muscle values of Gabriel et al. Similarly, the phase difference $\theta$ can be calculated by

$$
\theta=\arctan \left(\frac{J_{\text {disp }}}{J_{\text {cond }}}\right) \text {. }
$$

At $150 \mathrm{~Hz}$, this yields a phase difference of $32.0^{\circ}$. We see close agreement between these analytical estimations and the numerical FE calculations. These results indicate that displacement currents can be sizable in human muscle and have a significant impact on the electric potential distribution. They also demonstrate the accuracy of the finite element method. 
We chose dielectric values of Gabriel et al. [24] because it is the most recent and comprehensive work available. However, when surveying the literature, we see that Gabriel's estimates of permittivity and conductivity are fairly high. If we had used the Bodakian and Hart [21] values for muscle of $\varepsilon_{r}=3.7 \times 10^{6}$ and $\sigma=0.07 \mathrm{~S} / \mathrm{m}$ at $100 \mathrm{~Hz}$, the displacement currents would have been approximately $21 \%$ as large as the conduction current with a phase shift of $12^{\circ}$. At the other end of the spectrum, Epstein and Foster's [22] values of $\varepsilon_{r}=3.5 \times 10^{5}$ and $\sigma=0.08 \mathrm{~S} / \mathrm{m}$ yield displacement currents that are only about $2.4 \%$ as large as the conduction currents. Other tissues must be considered as well. Although the permittivity of skin $\left(\varepsilon_{r}=6.0 \times 10^{4}\right)$ is much lower than that of muscle at $100 \mathrm{~Hz}$, its conductivity is proportionately even lower $\left(\sigma=3.0 \times 10^{-4} \mathrm{~S} / \mathrm{m}\right)$ [24]. For this tissue, displacement currents would actually be larger than conduction currents (approximately $111 \%$ ). In summary, the capacitive properties of biological tissues are high and can significantly effect electromagnetic signal propagation. Further research on the effects of permittivity on bioelectric signal propagation is needed.

Our eventual goal is to use the FEM to quantify crosstalk and investigate ways to maximize signal independence. Perhaps the most important factor affecting fall-off of the electric potential field and, thus, crosstalk is how the source of the EMG signal is modeled. The simplest source model is a single dipole. It could be considered a worst case scenario for crosstalk because the fall-off of the electric field is fairly slow. This simple dipole is also important because it is a fair way to model the end effects of EMG signal propagation in muscle fiber. Although the crosstalk falls off rapidly with increasing angle around the limb, it remains significant (i.e., $>10 \%$ ) through an azimuth angle of over $45^{\circ}$, with this single point source at a depth of $10 \mathrm{~mm}$. The fall-off with the tripole and, most important, the realistic waveform sources at this depth were almost identical and were much faster than the dipole. These models indicate that significant EMG signal amplitude would be detected for about $30^{\circ}$ around the limb or a distance of $2.6 \mathrm{~cm}$. As larger and diffuse areas of muscle are activated, we would expect the angle of significant crosstalk to increase.

These models are quite simple and their limitations must be appreciated. Only a single source at a single depth studied. A real EMG is an accumulation of signals from hundreds of sources spread throughout a given volume of muscle. These models contain only a single tissue-muscle. The effects of fat, skin, and even bone also must be studied to understand how they affect EMG signal propagation and crosstalk. Finally, the effect of permittivity must be studied in greater detail and dispersion should be considered. Tissue permittivities can be very high and vary greatly with frequency. Little work has been done to understand the effects of permittivity and dispersion as it relates to bioelectric signal propagation.

Nevertheless, we believe these models demonstrate the usefulness of the FEM in studying EMG signal propagation. As we develop more complex models that incorporate multiple tissues, realistic geometries, and diffuse muscle activation, we will be able to accurately calculate EMG distributions, predict crosstalk, and study ways to increase myoelectric signal independence to improve the control of artificial limbs.

\section{ACKNOWLEDGMENT}

The authors would like to thank Dr. J. DeWald and Dr. C. J. Heckman for their generosity with their time and laboratory space and the use of their equipment.

\section{REFERENCES}

[1] J. A. Hoffer and G. E. Loeb, "Implantable electrical and mechanical interfaces with nerve and muscle," Ann. Biomed. Eng., vol. 8, pp. 351-360, 1980.

[2] C. J. De Luca and R. Merletti, "Surface myoelectric signal cross-talk amoung muscles of the leg," Electroenceph. Clin. Neurophys., vol. 69, pp. 568-575, 1988.

[3] T. Koh and M. D. Grabiner, "Cross talk in surface electromyograms of human hamstring muscles," J. Orthoped. Res., vol. 10, pp. 701-709, 1992.

[4] D. C. Boyd and P. D. Lawerence, "On modeling the single motor unit action potential," IEEE Trans. Biomed. Eng., vol. 25, pp. 236-243, May 1975.

[5] N. Dimitrova, "Model of the extracellular potential field of a single striated muscle fiber," Electroenceph. Clin. Neurophys., vol. 14, pp. 53-66, 1974.

[6] G. V. Dimitrov and N. A. Dimitrova, "Fundamentals of power spectra of extracellular potetntials produced by a skeletal muscle fiber of finite length part II: Effect of parameters altering with functional state," Med. Eng. Phys., vol. 20, pp. 702-702, 1998.

[7] J. Duchene and J. Y. Hogrel, "A model of EMG generation," IEEE Trans. Biomed. Eng., vol. 47, pp. 192-201, Febr. 2000.

[8] D. Farina and A. Rainoldi, "Compensation of the effect of sub-cutaneous tissue layers on surface EMG: A simulation study," Med. Eng. Phys., vol. 21, pp. 487-496, 1999.

[9] P. A. M. Griep, F. L. Gielen, K. L. Boon, L. L. Hoogstraten, C. W. Pool, and W. Wallinga-De Jonge, "Calculation and registration of the same motor unit action potential," Electroenceph. Clin. Neurophys., vol. 53, pp. 388-404, 1982.

[10] K. A. Henneberg and F. A. Roberge, "Simulation of propagation along an isolated skeletal muscle fiber in an isotropic volume conductor," Ann. Biomed. Eng., vol. 25, pp. 15-28, 1997.

[11] R. Merletti, L. L. Conte, E. Avignone, and P. Guglielminotti, "Modeling of surface myoelectric signals part I: Model implementation," IEEE Trans. Biomed. Eng., vol. 46, pp. 810-820, July 1999.

[12] B. Roth, F. Gielen, and J. Wiskwo, "Spatial and temporal frequency dependent conductivities in volume conduction calculations for skeletal muscle," Math. Biosci., vol. 88, pp. 159-189, 1988.

[13] N. A. Trayanova and G. V. Dimitrov, "Mathematical models of the extracellular potentials generated by excitable fibers in a volume conductor," Electromyogr. Clin. Neurophysiol., vol. 23, pp. 123-137, 1983.

[14] N. Ganapathy, J. W. Clark, and O. B. Wilson, "Extracellular potentials form skeletal muscle," Math. Biosci., vol. 83, pp. 61-96, 1987.

[15] D. A. Winter, A. J. Fuglevand, and S. E. Archer, "Crosstalk in surface electromyography: Theoretical and practical estimates," J. Electromyography Kinesiol., vol. 4, pp. 15-26, 1994.

[16] T. H. Gootzen, D. F. Stegeman, and A. van Oosterom, "Finite limb dimensions and finite muscle length in a model for the generation of electromyographic signals," Electroenceph. Clin. Neurophys., vol. 81, pp. $152-162,1991$.

[17] K. A. Henneberg and R. Plonsey, "Boundary element analysis of the directional sensitivity of the concentric EMG electrode," IEEE Trans. Biomed. Eng., vol. 40, pp. 621-631, July 1993.

[18] K. McGill and A. Huynh, "A model of the surface recorded motor unit action potential," in Proc. 10th Аnnu. Int. IEEE Conf. Engineering Medicine Biology, 1988, pp. 1697-1699.

[19] K. Roeleveld, J. Blok, D. Stegeman, and A. V. Oosterom, "Volume conduction models for surface EMG: Confrontation with measurements," J. Electromyography. Kinesiol., vol. 7, pp. 221-232, 1997.

[20] B. K. van Veen, N. J. Rijkhoff, W. L. Rutten, W. Wallinga, and H. B. Boom, "Potential distribution and single-fiber action potentials in a radially bounded muscle model," Med. Biol. Eng. Comput., vol. 30, pp. 303-310, 1992.

[21] B. Bodakian and F. X. Hart, "The dielectric properties of meat," IEEE Trans. Dielect. Elect. Insulation, vol. 1, pp. 181-187, 1994.

[22] B. R. Epstein and K. R. Foster, "Anisotropy in the dielectric properties of skeletal muscle," Med. Biol. Eng. Comput., vol. 21, pp. 51-55, 1983.

[23] S. Gabriel, R. W. Lau, and C. Gabriel, "The dielectric properties of biological tissues: I. Literature survey," Phys. Med. Biol., vol. 41, pp. 2231-2249, 1996 
[24] _ _ "The dielectric properties of biological tissues: II. Measurements in the frequency range $10 \mathrm{~Hz}$ to $20 \mathrm{GHz}$," Phys. Med. Biol, vol. 41, pp. 2251-2269, 1996.

[25] — "The dielectric properties of biological tissues: III. Parametric models for the dielectric spectrum of tissues," Phys. Med. Biol, vol. 41, pp. 2271-2293, 1996.

[26] B. A. Alber, W. L. Rutten, W. Wallinga-de Jonge, and H. B. Boom, "Frequency domain modeling of volume conduction of single muscle fiber action potentials," IEEE Trans. Biomed. Eng., vol. 35, pp. 328-332, May 1988.

[27] W. L. Rutten, B. K. van Veen, S. H. Stroeve, H. B. Boom, and W. Wallinga, "Influence of inhomogeneities in muscle tissue on single-fiber action potentials: A model study," Med. Biol. Eng. Comput., vol. 35, pp. 91-95, 1997.

[28] R. Plonsey, "The active fiber in a volume conductor," IEEE Trans. Biomed. Eng., vol. BME-21, pp. 371-381, 1974.

[29] N. A. Trayanova, C. S. Henriquez, and R. Plosey, "Limitation of approximate solutions for computing the extracellular potential of single fibers and bundle of equivalents," IEEE Trans. Biomed. Eng., vol. 37, pp. 22-34, Jan. 1990.

[30] P. Rosenfalck, "Intra- and extracellular potential fields of active nerve and muscle fibers. A physcio-mathematical analysis of different models," Acta Physiol. Scand., vol. supp. 321, pp. 1-168, 1969.

[31] S. Nandedkar and E. Stalberg, "Simulation of single muscle fiber action potentials," Med. Biol. Eng. Comput., vol. 21, pp. 158-165, 1983.

[32] K. Roeleveld, D. Stegeman, H. Vingerhoets, and A. van Oosterom, "Motor unit potential contribution to surface electromyography," Acta Physiol. Scand., vol. 160, pp. 175-183, 1997.

[33] - "The motor unit potential distribution over the skin surface and its use in estimating the motor unit location," Acta Physiol. Scand., vol. 161, pp. 465-472, 1997.

[34] R. Plonsey and D. B. Heppner, "Considerations of quasistationarity in electrophysiologicaal systems," Bull. Math. Biophys., vol. 29, 1967.

Todd A. Kuiken (M'00) received the Ph.D. degree in biomedical engineering and the M.D. degree from Northwestern University, Evanston, IL, in 1989 and 1990, respectively.

He was trained in physical medicine and rehabilitation at the Rehabilitation Institute of Chicago, Chicago, IL, and is a board-certified physiatrist. He is currently Chief of Staff and the Director of Amputee Services at the Rehabilitation Institute of Chicago. He is an Assistant Professor in the Department of Physical Medicine and Rehabilitation and the Electrical and Computer Engineering Department of Northwestern University. His research interests include the care of amputees, the control of artificial limbs, and myoelectric signal analysis.

Nikolay S. Stoykov (M'00) received the M.S. and Ph.D. degrees in biomedical engineering from the Technical University Ilmenau, Ilmenau, Germany, in 1990 and 1998, respectively.

Since 1999, he has been a Postdoctoral Fellow with the Department of Research, Rehabilitation Institute of Chicago, Chicago, IL, and with the Department of Physical Medicine and Rehabilitation, Northwestern University, Evanston, IL. His research interests have focused on modeling bioelectric phenomena with FEM.
Milica Popović (S'92-M'01) received the B.S. degree in electrical engineering from University of Colorado, Boulder, in 1994. She received the M.S. and Ph.D. degrees in electrical engineering from Northwestern University, Evanston, IL, in 1997 and 2001, respectively.

In 2001, she joined the Department of Electrical and Computer Engineering, McGill University, Montreal, QC, Canada. Her current research interests include computational electromagnetics, inverse problems, electromagnetic applications in medicine and biology, and antenna design.

Dr. Popović is a member of Eta Kappa Nu, the IEEE Antennas and Propagation Society, the IEEE Microwave Theory and Techniques Society, and the Bioelectromagnetics Society.

Madeleine Lowery (M'01) received the B.E. and Ph.D. degrees from the Department of Electronic and Electrical Engineering, University College Dublin, National University of Ireland,Dublin, in 1996 and 2000, respectively.

She is currently a Postdoctoral Research Fellow with the Department of Physical Medicine and Rehabilitation, Rehabilitation Institute of Chicago, Chicago, IL. Her research is focused on mathematical modeling and analysis of bioelectric signals, particularly surface EMG.

Allen Taflove (M'75-SM'84-F'90) was born in Chicago, IL, on June 14, 1949. He received the B.S., M.S., and Ph.D. degrees in electrical engineering from Northwestern University, Evanston, IL, in 1971, 1972, and 1975, respectively.

In 1984, he returned to Northwestern University after nine years as a Research Engineer at IIT Research Institute, Chicago, IL. Since 1988, he has been a Professor in the Department of Electrical and Computer Engineering, McCormick School of Engineering, Evanston, IL. Since 1972, he has pioneered basic theoretical approaches and engineering applications of finite-difference time-domain (FDTD) computational electromagnetics. He coined the FDTD acronym in a 1980 IEEE paper. In 1995, he authored Computational Electrodynamics: The Finite-Difference Time-Domain Method. This book is now in its second edition, coauthored with Prof. Susan Hagness of the University of Wisconsin, Madison. In 1998, he was the editor of the research monograph, he has authored or coauthored 12 invited book chapters, 73 journal papers, and approximately 200 conference papers and abstracts, and he holds 13 U.S. patents. He has been the thesis adviser for $14 \mathrm{Ph}$.D. recipients who hold professorial, research, or engineering positions at major institutions, including the University of Wisconsin, Madison, the University of Colorado, Boulder, McGill University, Montreal, QC, Canada, Lincoln Laboratories, Jet Propulsion Laboratories, and the U.S. Air Force Research Lab. Currently, he is conducting research in a wide range of computational electromagnetics modeling problems, including the propagation of bioelectric signals within the human body, laser-beam propagation within samples of human blood, ultrahigh frequency (UHF) diffraction by buildings in urban wireless microcells, microwave cavity resonances in subatomic particle accelerators, electrodynamics of micron-scale optical devices, novel wireless interconnects for ultrahigh-speed digital data buses, and extremely low frequency (ELF) geophysical phenomena.

In 1990, Dr. Taflove was the first person to be named a Fellow of the IEEE in area of FDTD. Currently, he is a Charles Deering McCormick Professor of Teaching Excellence and Master of the Lindgren Residential College of Science and Engineering. 